\title{
Nucleon-nucleus optical potential in the particle-hole approach
}

\author{
C. Barbieri* and B. K. Jennings \\ TRIUMF, 4004 Wesbrook Mall, Vancouver, British Columbia, Canada V6T 2A3 \\ (Received 28 January 2005; published 29 July 2005)
}

\begin{abstract}
Feshbach's projection formalism in the particle-hole model space leads to a microscopic description of scattering in terms of the many-body self-energy. To investigate the feasibility of this approach, an optical potential for ${ }^{16} \mathrm{O}$ is constructed starting from two previous calculations of the self-energy for this nucleus. The results reproduce the background phase shifts for positive parity waves and the resonances beyond the mean field. The latter can be computed microscopically for energies of astrophysical interest using Green's function theory.
\end{abstract}

DOI: 10.1103/PhysRevC.72.014613

PACS number(s): 24.10.Cn, 25.40.Cm, 25.40.Lw, 21.10.Jx

\section{INTRODUCTION}

The Feshbach's theory of the optical potential [1] provides a tool to describe the scattering of nucleons from nuclei. In its original form, the full Hilbert space is partitioned in a subspace that contains only one particle added to a particular state of the core nucleus. This includes the elastic scattering states and the nuclear orbits unoccupied by the target's nucleons. The resulting optical potential can be thought as an effective interaction that accounts for the effects of the degrees of freedom of the excluded space - such as the overall antisymmetrization of the wave function, excitations of the target, or breakup channels. The complexity of these effects makes the $a b$ initio calculation of the optical potential a very difficult task. The choice of working in the space of one particle plus a core is shared by several theories of nucleon-nucleus scattering. Examples are the cluster model [2], folding potential [3], and shell model embedded in the continuum [4]. All these techniques have been successfully applied to scattering processes.

A conceptually different approach consists in extending the scattering space to include both a particle on top of the nuclear core and the possibility of propagating a hole excitation. In the following, we will refer to this as the particle-hole (ph) Hilbert space. Mahaux and Capuzzi [5] and Jennings and Escher [6] have shown that applying the Feshbach's formalism to this space leads to an optical potential that is the usual many-body self-energy defined in Green's function theory $[7,8]$. The properties of using the self-energy as an optical potential have been discussed by Mahaux and Sartor in Ref. [9]. The solutions of the scattering equation above (below) the Fermi energy are the overlap wave functions between the core and the eigenfunctions of the systems with $A+1(A-1)$ nucleons. For particle states these are the same wave functions as obtained in the original Feshbach approach [1]. The fact that states with $A-1$ particles are included in the formalism does not lead to complications in the study of scattering events. Rather, more physical information can be extracted using the self-energy since it also describes the so-called "Pauli forbidden" orbitals, occupied by the nucleons of the target. Correspondingly, the optical potential in the ph space is

*Electronic address: barbieri@triumf.ca more easily comparable to phenomenological models (based on, e.g., Woods-Saxon wells) [5,9] since these also describe hole states. It also has better analytical properties than its counterpart in the particle-only space [5,6]. Finally, the theory of Green's functions provides a natural way to include the effects of the excitations of the core in terms of an expansion in Feynman diagrams $[9,10]$.

Many-body Green's functions have been applied in the past to study nuclear correlations, with emphasis on the hole part of the one-body spectral function. Recent developments offer the opportunity to obtain sophisticate descriptions of the couplings between particles and collective states using a Faddeev expansion [11,12]. A similar formalism was already considered in Ref. [13] for the optical potential where, however, the dressing of propagators was disregarded and no application was attempted. The calculations of Refs. [11,14] account for collective motion near the Fermi level, including the energy regime of interest to nuclear astrophysics. With a variety of exotic isotopes that are involved in stellar processes becoming experimentally accessible in modern radioactive beam facilities, it is important to investigate whether the Green's function approach can be applied to study low-energy nucleon scattering and capture processes.

We have recently considered the self-energy resulting from a recent application of the self-consistent Green's function (SCGF) method to ${ }^{16} \mathrm{O}$ [14] and explored its predictions for proton-nucleus scattering. These results were obtained in a restricted model space, which is not fully appropriate to describe scattering events. However, preliminary calculations with only this input gave encouraging results [15]. In this work the self-energy of Ref. [14] is augmented by including the components from outside this model space, as they have been computed in Ref. [16]. We then report on the final conclusions of these exploratory studies.

The model and the details of the calculations are given in Sec. II. The results for elastic scattering and the bound states of ${ }^{17} \mathrm{~F}$ are reported in Sec. III and are preceded by a discussion of the different contributions to the self-energy, in Sec. III A. A discussion and our conclusion are given in Secs. IV and V.

\section{MODEL}

In the SCGF approach it is useful to split the manybody self-energy into three contributions [10,17], as shown 


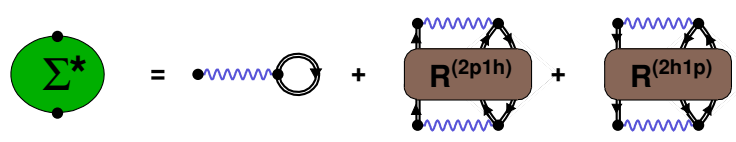

FIG. 1. (Color online) Feynman diagrams representation of the self energy. The first diagram on the right-hand side represents the Hartree-Fock-like contribution to the mean field. The remaining ones describe core polarization effects in the particle $(2 \mathrm{p} 1 \mathrm{~h})$ and hole (2h1p) part of the spectrum.

diagrammatically in Fig. 1. There, the double lines represent the exact one-body Green's function, which contains complete information on the particle and hole spectral distributions. The first diagram on the right-hand side is the direct extension of the Hartree-Fock potential to include the effects of the fragmentation of strength and represents the nuclear mean-field (MF) in the presence of correlations. The remaining contributions split naturally in diagrams containing at least two-particle-one-hole (2p1h), describing the system of $A+1$ particles, or two-hole-one-particle $(2 \mathrm{~h} 1 \mathrm{p})$, corresponding to $A-1$ particles. The irreducible propagators $R^{(2 \mathrm{plh})}$ and $R^{(2 \mathrm{~h} 1 \mathrm{p})}$ account for the core polarization contributions to the optical potential in the particle and hole spaces, respectively [9]. The separation of Fig. 1 is exact. In Refs. [11,14], $R^{(2 \mathrm{plh})}$ and $R^{(2 \mathrm{~h} 1 \mathrm{p})}$ were computed employing a Faddeev expansion that permits the direct coupling of the single-particle motion to collective excitations of the core. These were evaluated in the dressed random phase approximation (DRPA) [18]. The example of a diagram that contributes to $R^{(2 \mathrm{plh})}$ is given in Fig. 2. Since this expansion is based on the fully fragmented single particle propagator - which is generated from the self-energy itselfa self-consistent solution is required.

The nuclear self-energy computed in Ref. [14] was obtained within a model space $\mathcal{P}$ corresponding to the harmonic oscillator wave functions for all orbitals up to the $p f$ shell plus the $g_{9 / 2}$. A parameter $b=1.76 \mathrm{fm}$ was employed. This space

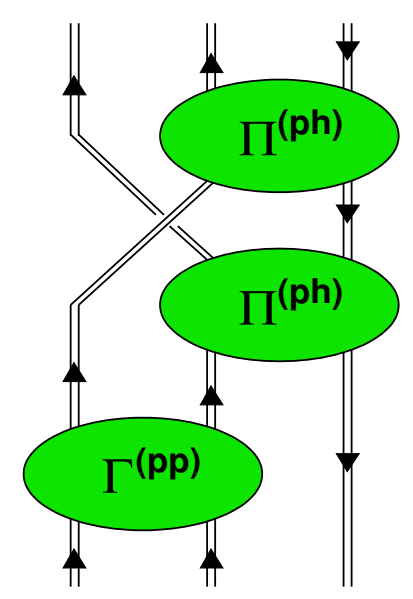

FIG. 2. (Color online) Example of a diagrammatic contribution included in the Faddeev expansion for $R^{(2 \mathrm{plh})}$ (see Fig. 1). A quasiparticle is coupled to the response function $\Pi^{(\mathrm{ph})}$ that describes the target nucleus. It can also participate in pairing processes, which are accounted for by the two-body propagator $g^{I I,(\mathrm{pp})}$. appears to be large enough to describe the influence of the low energy (long-range) excitations on nuclear fragmentation [12]. However, it requires a proper extension for applications to single particle scattering, as it will be discussed below. The effect of correlations outside this model space were accounted for by employing a $G$ matrix as an effective interaction, which was derived from the Bonn-C potential [19] according to Ref. [20]. The computation of the $G$ matrix for positive energies is an outstanding problem which was not attempted there. Therefore, we employed a fixed starting energy of $-5 \mathrm{MeV}$ in the present work, as the closest reliable choice to the continuum.

At low energies the optical potential is well approximated by a real interaction and $R^{(2 \mathrm{p} 1 \mathrm{~h})}$ and $R^{(2 \mathrm{~h} 1 \mathrm{p})}$ can be expressed as discrete sums of poles. Thus, for each given partial wave, $l j$, the contributions depicted in Fig. 1 can be expressed as

$$
\begin{aligned}
\Sigma_{l j}^{\mathrm{MF}, \text { Fadd }}\left(k, k^{\prime}\right) & =\sum_{n_{\alpha}, n_{\beta} \in \mathcal{P}} \phi_{\alpha}(k) \Sigma_{l j ; n_{\alpha}, n_{\beta}}^{\mathrm{MF}, \text { add }} \phi_{\beta}^{*}\left(k^{\prime}\right), \\
\Sigma_{l j}^{(2 \mathrm{p} 1 \mathrm{~h}), \text { Fadd }}\left(k, k^{\prime}\right) & =\sum_{n_{\alpha}, n_{\beta} \in \mathcal{P}} \phi_{\alpha}(k)\left[\sum_{n+} \frac{\left(m_{\alpha}^{n+}\right)^{*} m_{\beta}^{n+}}{\omega-\varepsilon_{l j}^{n+}+i \eta}\right] \phi_{\beta}^{*}\left(k^{\prime}\right), \\
\Sigma_{l j}^{(2 \mathrm{~h} 1 \mathrm{p}), \text { Fadd }}\left(k, k^{\prime}\right) & =\sum_{n_{\alpha}, n_{\beta} \in \mathcal{P}} \phi_{\alpha}(k)\left[\sum_{k-} \frac{\left(m_{\alpha}^{k-}\right)^{*} m_{\beta}^{k-}}{\omega-\varepsilon_{l j}^{k-}-i \eta}\right] \phi_{\beta}^{*}\left(k^{\prime}\right),
\end{aligned}
$$

where $\phi_{\alpha}(r)$ are the harmonic oscillator radial functions referring to single particle quantum numbers $\alpha=\left\{n_{\alpha}, l_{\alpha}, j_{\alpha}, m_{\alpha}\right\}$ [38], the first sum runs over all the orbits belonging to the model space and $l_{\alpha} j_{\alpha}=l_{\beta} j_{\beta}=l j$ since ${ }^{16} \mathrm{O}$ has a $0^{+}$isoscalar ground state.

The superscript "Fadd" indicates that Eqs. (1) represent the results of Ref. [14]. This is the most sophisticated calculation available to date for the self-energy at low-energies that account for the coupling between single nucleons and collective excitations. However, the expansion over a few harmonic oscillator states is not optimal for describing the details of the nuclear surface. Analogously, it misses part of the large momentum components in the optical potential. This is particularly critical for the MF component, which describes the background of the phase shifts. On the contrary, the same nucleus was studied in Ref. [16] employing a spherical box basis that includes all the relevant momentum components. An effective $G$ matrix, derived for nuclear matter and the Bonn-B potential [19], accounted for the binding due to short-range and tensor correlations. The self-energy, computed only to second order in the perturbation series, neglected most of the collective effects. This approach was applied to obtain the quasihole wave functions associated to the $p$ states occupied in ${ }^{16} \mathrm{O}$, with sufficiently accurate results to describe the shapes of the $\left(e, e^{\prime} p\right)$ cross sections to those states [21].

In this work, we chose to employ a mixed representation of the self-energy in which the MF components missing in the space $\mathcal{P}$ were extracted from Ref. [16], while the contributions beyond MF computed in Ref. [14] [Eqs. (1b) and (1c)] were 
retained. To do this the MF self-energy of Ref. [16] was split in two parts:

$$
\Sigma_{l j}^{\mathrm{MF}, \mathrm{Box}}\left(k, k^{\prime}\right)=\Sigma_{0, l j}^{\mathrm{MF}, \mathrm{Box}}\left(k, k^{\prime}\right)+\Sigma_{1, l j}^{\mathrm{MF}, \mathrm{Box}}\left(k, k^{\prime}\right),
$$

where $\Sigma_{0}^{\mathrm{MF}, \mathrm{Box}}$ is the projection onto $\mathcal{P}$ and $\Sigma_{1}^{\mathrm{MF}, \mathrm{Box}}$ acts on the excluded space. Two approximations were considered depending on which MF component to employ inside $\mathcal{P}$. In the first case (I), $\Sigma_{1}^{\mathrm{MF}, \mathrm{Box}}$ was added to Eq. (1a). In doing this, we note that the $G$ matrix used to compute $\Sigma^{\mathrm{MF} \text {,Fadd }}$ accounts for the extra binding due to the degrees of freedom of the excluded space. Since these are reinserted explicitly by $\Sigma_{1}^{\mathrm{MF} \text {,Box }}$, one should also rescale $\Sigma^{\mathrm{MF} \text {,Fadd }}$ appropriately by a constant, $N^{I}$. The second choice (II) consisted of employing both parts of Eq. (2). Also in this case we kept the possibility of tuning the depth of the potential. The complete MF contributions employed in this work are

$$
\begin{aligned}
\Sigma_{l j}^{\mathrm{MF}, I}\left(k, k^{\prime}\right) & =N_{l j}^{I} \Sigma_{l j}^{\mathrm{MF}, \text { Fadd }}\left(k, k^{\prime}\right)+\Sigma_{1, l j}^{\mathrm{MF}, \text { Box }}\left(k, k^{\prime}\right), \\
\Sigma_{l j}^{\mathrm{MF}, I I}\left(k, k^{\prime}\right) & =N_{l j}^{I I} \Sigma_{l j}^{\mathrm{MF}, \mathrm{Box}}\left(k, k^{\prime}\right),
\end{aligned}
$$

where the constants $N_{l j}^{I}$ and $N_{l j}^{I I}$ depend of the specific channel and will be discussed below. The full self-energy employed in the calculations is (see Fig. 1)

$$
\begin{aligned}
\Sigma_{l j}^{\star, I(I I)}\left(k, k^{\prime} ; \omega\right)= & \Sigma_{l j}^{\mathrm{MF}, I(I I)}\left(k, k^{\prime}\right)+\Sigma_{l j}^{(2 \mathrm{p} 1 \mathrm{~h}), \text { Fadd }}\left(k, k^{\prime} ; \omega\right) \\
& +\Sigma_{l j}^{(2 \mathrm{~h} 1 \mathrm{p}), \text { Fadd }}\left(k, k^{\prime} ; \omega\right) .
\end{aligned}
$$

The Dyson equation can be expressed in a Schrödinger-like form, where the self-energy takes the place of a nonlocal and energy dependent optical potential $[\hbar=c=1$ and $\mu$ is the reduced mass]

$$
\begin{gathered}
\frac{k^{2}}{2 \mu} \psi(k)+\int_{0}^{\infty} d k^{\prime} k^{\prime 2}\left\{\Sigma_{l j}^{\star}\left(k, k^{\prime} ; E_{\text {c.m. }}\right)+V_{\text {Coul. }}^{l}\left(k, k^{\prime}\right)\right\} \psi\left(k^{\prime}\right) \\
=E_{\text {c.m. }} \psi(k),
\end{gathered}
$$

where $V_{\text {Coul. }}^{l}\left(k, k^{\prime}\right)$ in the Coulomb interaction corresponding to a uniformly charged sphere of radius $R_{c}=3.1 \mathrm{fm}$. This was added to account for the electromagnetic interaction missing in the calculations of Refs. [14,16]. Due to the nonlocal character of $\Sigma^{\star}$, Eq. (5) is conveniently solved in momentum space. In doing this, the long distance part of the Coulomb potential was solved using the Kwon-Tabakin-Lande [22] procedure for bound states and the Vincent-Phatak [23] one for scattering.

Above the Fermi level the eigenvalues of Eq. (5) are related to the spectrum of ${ }^{17} \mathrm{~F}$ by $E_{\text {c.m. }}^{n}=E_{n}^{17} \mathrm{~F}-E_{\text {g.s. }}^{16}$. Thus, $E_{\text {c.m. }}>0$ describes the scattering of protons from ${ }^{16} \mathrm{O}$ while the bound solutions are the overlaps of the ground state of ${ }^{16} \mathrm{O}$ with the corresponding bound states ${ }^{17} \mathrm{~F}$. Analogously, below the Fermi level $E_{\text {c.m. }}^{n}=E_{\mathrm{g} . \mathrm{S} .}^{160}-E_{n}^{15} \mathrm{~N}$ and the eigenstates represent the overlaps with ${ }^{15} \mathrm{~N}$. The Dyson equation implies that the bound solutions of Eq. (5) have to be normalized to their spectroscopic factor according to

$Z_{l j}^{n}=\int_{0}^{\infty} d k k^{2}\left|\psi^{n}(k)\right|^{2}=\left[1-\left.\left\langle\tilde{\psi}^{n}\left|\frac{d \Sigma_{l j}^{\star}}{d \omega}\right| \tilde{\psi}^{n}\right\rangle\right|_{\omega=E_{\mathrm{c} . \mathrm{m} .}^{n}}\right]^{-1}$, where $\tilde{\psi}^{n}(k)$ is the solution itself normalized to unity and $E_{\mathrm{c.m}}^{n}$. is the corresponding eigenvalue. The asympotic normalization for the unbound solutions is related in the usual way to the flux of incoming particles.

\section{RESULTS}

Equations (3) and (4) include the relevant physics from both the calculations of Refs. [14] and [16]. This self-energy represents a model for the optical potential that acts on the full ph Hilbert space and can give sensible predictions near the Fermi level. However, the two-body realistic interactions alone, as used in these works, cannot reproduce the experimental binding energies and spin-orbit splitting for nuclei with $A \geqslant 3[24,25]$. To obtain these, relativistic effects or three-body forces are required [26]. In this work $\Sigma^{\star}$ was constrained to reproduce the experimental spectrum in two ways. First, the constants $N_{l j}^{I}$ and $N_{l j}^{I I}$ that affect the depth of the optical potential were chosen to reproduce the corresponding quasiparticle energies. These are the $s_{1 / 2}$ and $d_{5 / 2}$ bound states of ${ }^{17} \mathrm{~F}$, its $d_{3 / 2}$ resonance and the $p_{1 / 2}$ and $p_{3 / 2}$ hole states of ${ }^{15} \mathrm{~N}$. Second, complex resonances that do not have a mean field character are generated by the dynamic part of the self-energy. At low energy, most of these couple to only one pole $\varepsilon^{i \pm}$ in Eqs. (1b) and (1c). Therefore, we have fitted those poles that could be identified with specific resonances of the $A+1$ system $\left({ }^{17} \mathrm{~F}\right)$ by imposing that Eq. (5) yields the corresponding experimental energies. We note that a similar approach was already employed in Ref. [14]. This is necessary for the particular case of ${ }^{16} \mathrm{O}$ due to the strong coupling between the single particle spectrum and collective motions, which suggest the need for an improved description of the low-energy structure of this nucleus [12] and more attractive effective interactions [27]. Although, satisfactory results can already be obtained in similar calculations for heavier nuclei [28-30].

The influence of this fitting procedure on the results is discussed in the following. After calibrating Eqs. (1) and (3) to the spectra of ${ }^{17} \mathrm{~F}$ and ${ }^{15} \mathrm{~N}$, the results for the scattering phase shifts and the bound single particle wave functions are a prediction of the model.

\section{A. Parameter dependence}

To discuss the influence of the different contributions to Eq. (3), the phase shifts for proton scattering have been computed employing different truncations of the mean field self-energy $\Sigma^{\mathrm{MF}, I}$. The results are shown in Fig. 3 for three partial waves. The dotted lines were obtained by retaining only the original contribution to the self-energy of Ref. [14]. Thus, neglecting $\Sigma_{1}^{\mathrm{MF}, \text { Box }}$ in Eq. (3a) and setting $N_{l j}^{I}=1$ for all cases. The results obtained by constraining these constants to generate the proper quasiparticle energies is given by the dashed lines. The full line shows the full results form Eq. (3a), obtained by including also the $\Sigma_{1}^{\mathrm{MF}, \mathrm{Box}}$ term and refitting the $N_{l j}^{I}$. The values for the quasiparticle energies and the constants $N_{l j}^{I}$ used are given in Table I. 


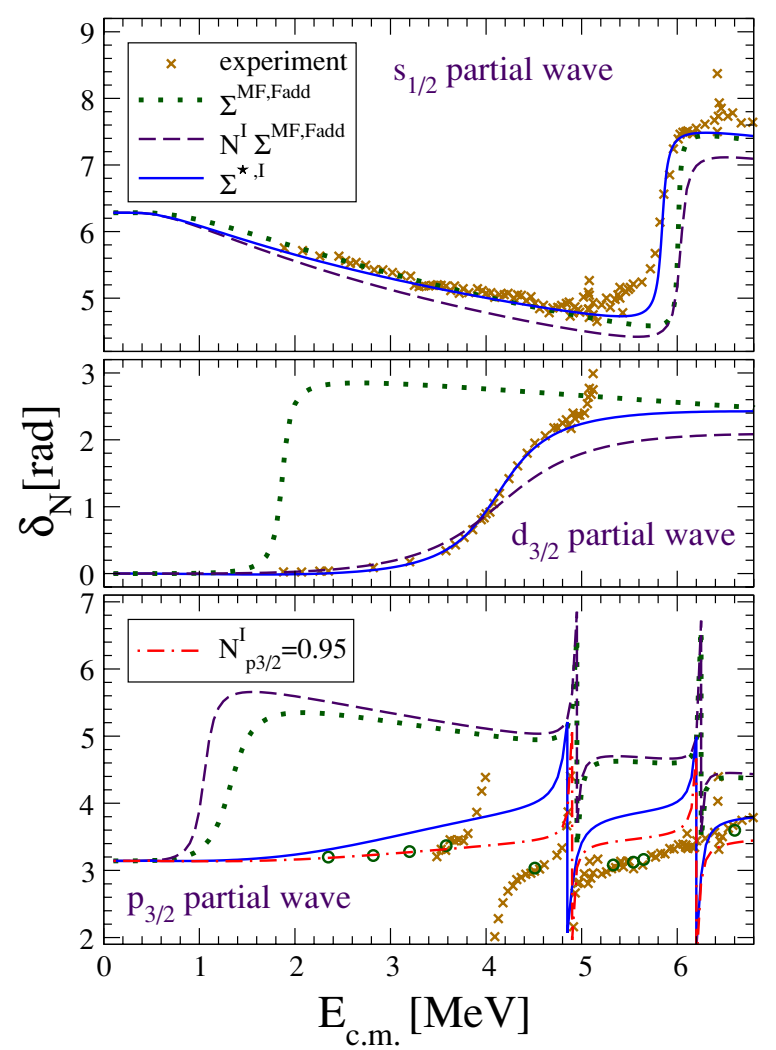

FIG. 3. (Color online) Phase shifts obtained from the selfconsistent self-energy of Ref. [14] [Eq. (1)] before (dotted lines) and after (dashed lines) correcting the depth of $\Sigma^{\mathrm{MF} \text {,Fadd }}$ to reproduce the quasiparticle (and quasihole) energies. The full lines are obtained also when the momentum components outside the model space $\mathcal{P}$ are included, Eq. (3a). The $s_{1 / 2}, d_{3 / 2}$, and $p_{3 / 2}$ partial waves are shown. For $p_{3 / 2}$, the dot-dashed line was obtained by fitting $N_{p_{3 / 2}}^{I}$ to reproduce the background phase shifts rater than the quasihole energy. The values of the corrections $N_{l j}^{I}$ are reported in Table I. The experimental results are from Refs. [31] (crosses) and [32] (circles).

The background contribution to the phase shifts of the $s_{1 / 2}$ partial wave is described correctly by $\Sigma^{\mathrm{MF} \text {,Fadd }}$ but not the energy of the bound state. Vice versa, it is possible to constrain the depth of the potential to reproduce the latter but the agreement with the experimental phase shifts is

TABLE I. Corrections applied to the depth of the MF potential $\Sigma^{\mathrm{MF}, I}[\mathrm{Eq}$. (3a)] and quasiparticle energies obtained in the calculations of Fig. 3.

\begin{tabular}{lcccr}
\hline \hline$l j$ & $\Sigma^{\mathrm{MF}, \text { Fadd }}$ & $\Sigma^{\mathrm{MF}, \text { Fadd }}$ & $\Sigma^{\star, I}$ & $E_{\mathrm{c} . \mathrm{m} .}^{\exp }(\mathrm{MeV})$ \\
& $E_{\mathrm{c} . \mathrm{m} .}^{l j}(\mathrm{MeV})^{\mathrm{a}}$ & $N_{l j}^{I \mathrm{~b}}$ & $N_{l j}^{I \mathrm{~b}}$ & \\
\hline$s_{1 / 2}$ & -3.57 & 0.69 & 1.05 & -0.1 \\
$d_{3 / 2}$ & 1.87 & 0.72 & 1.08 & 4.4 \\
$p_{3 / 2}$ & -16.61 & 1.06 & 1.07 & -18.5 \\
& & & $0.95\left(E_{\mathrm{c} . \mathrm{m} .}^{p_{3}}=-15.1\right)$ & \\
\hline \hline
\end{tabular}

${ }^{\mathrm{a}} N_{l j}^{I}=1$.

${ }^{\mathrm{b}} E_{\mathrm{c} . \mathrm{m} .}^{l j} \equiv E_{\mathrm{c} . \mathrm{m} .}^{\mathrm{exp}}$, except when specified. lost. However, both quantities are reproduced if $\Sigma_{1}^{\mathrm{MF}, \mathrm{Box}}$ is included. In this case the correction required in the depth of the potential, $N_{S_{1 / 2}}^{I}=1.05$, is less significant than when only $\Sigma^{\mathrm{MF} \text {,Fadd }}$ is included. A similar trend is seen for the $d_{3 / 2}$ channel. Reproducing the energy of the single particle resonance with $\Sigma^{\mathrm{MF} \text {,Fadd }}$ alone requires a sizable change in its depth, while the observed phase shifts are obtained only after including the components outside the space $\mathcal{P}$. We observe that the expansion of Eq. (1) includes only one harmonic oscillator function for the $d_{3 / 2}$ wave and two for $s_{1 / 2}$. With such a restricted space, it is remarkable that the resulting background phase shifts are still obtained somewhat close to the experiment.

A different behavior is found for the $l=1$ partial waves. The results for $p_{3 / 2}$ are shown in Fig. 3 (the $p_{1 / 2}$ case is analogous). In this case $\Sigma^{\mathrm{MF} \text {,Fadd }}$ produces a spurious resonance at $\sim 1 \mathrm{MeV}$ that is not seen experimentally. Fitting the potential's depth to constrain the quasihole energies of ${ }^{15} \mathrm{~N}$ generates a more attractive well, thus worsening the situation. The phase shifts improve upon introducing $\Sigma_{1}^{\mathrm{MF}, \text { Box }}$ (full line) but still show a rise of the background with the c.m. energy, while the experimental results are practically constant. A proper choice of $N_{p_{3 / 2}}^{I}$ (and $N_{p_{1 / 2}}^{I}$ ) allows to reproduce the behavior of the phase shifts at the lower energies but results in underbinding the corresponding orbitals in ${ }^{16} \mathrm{O}$ (see Table I).

The curves of Fig. 3 have been computed without any shift of the $\varepsilon^{i+}$ poles in in Eq. (1b). This gives an idea of the quality the energy spectra obtained adopting the interaction of Ref. [20]. No solutions were obtained that could be interpreted as the $d_{3 / 2}$ resonances above $5 \mathrm{MeV}$. The $s_{1 / 2}$ resonance at $\sim 6 \mathrm{MeV}$ was obtained as a coupling of a proton to the first excited state of ${ }^{16} \mathrm{O}$. Analogously, the two lowest resonances in both $p_{1 / 2}$ and $p_{3 / 2}$ can be interpreted as quasiparticles interacting with the first isoscalar $3-$ and $1-$ levels of ${ }^{16} \mathrm{O}[33]$.

\section{B. Phase shifts for proton scattering}

Figures 4 and 5 compare the phase shifts obtained from both the self-energies I and II after constraining the quasiparticle energies and resonances to their experimental values. Table II shows the values of the constants $N_{l j}^{I}$ and $N_{l j}^{I I}$ used to obtain these results.

For the positive parity waves the background phase shifts are described equally well by both optical potentials. The potential $\Sigma^{\star, I I}$ can also describe the negative parity waves and it is more accurate for the $p_{1 / 2}$ case, for which the collective resonances are sharper. In general, the non-MF resonances were predicted narrower than the experiment. This is probably related to the lack of momentum components outside the model space $\mathcal{P}$ in Eqs. (1b) and (1c), which were not corrected as for the MF part of the self-energy.

The values of $N_{l j}^{I}$ and $N_{l j}^{I I}$ show that much smaller modifications are needed to force $\Sigma^{\star, I}$ to reproduce the quasiparticle energies. This is consistent with the more sophisticate treatment of long-range correlations achieved in 


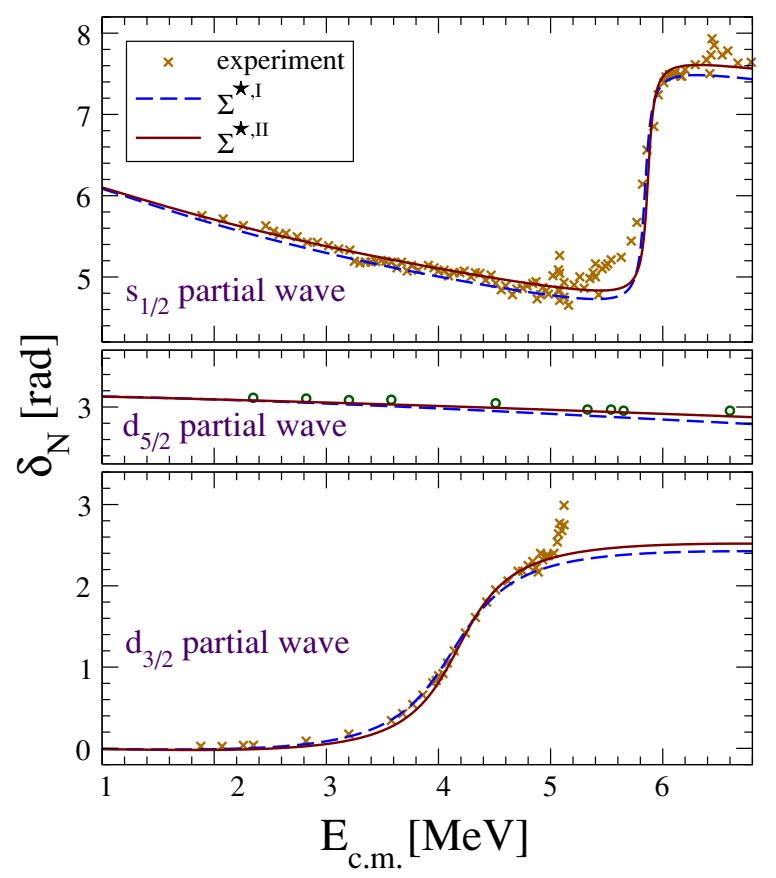

FIG. 4. (Color online) Phase shifts for positive parity waves obtained from the self-energies I (dashed lines) and II (full lines). The experimental results are from Refs. [31] (crosses) and [32] (circles).

Ref. [14]. It is worth noting that the inability to describe both the bound energies and the scattering for the negative parity waves is consistent with the lack of three-body forces in the present model, which are needed to reproduce their spin-orbit splitting. On the other hand the results with the MF potential II reproduce reasonably well both the quasihole energies and the phase shifts.

\section{Bound overlap wave functions}

The overlap wave functions associated to the bound states of ${ }^{17} \mathrm{~F}$ are shown in Fig. 6 for the two choices of Eq. (3). The asymptotic behavior in presence of a Coulomb field is given by

$$
\psi_{l j}(r) \longrightarrow_{r \rightarrow \infty} C_{l j} \frac{W_{-\eta, l+1 / 2}(r)}{r},
$$

where $W_{-\eta, l+1 / 2}$ is a Whittaker function, $\eta$ the Sommerfield parameter, and $C_{l j}$ the asymptotic normalization constant (ANC). The spectroscopic factors, ANCs and root-meansquare radii obtained are given in Table III.

The self-energy $\Sigma^{\star, I}$ predicts larger radii and ANCs than $\Sigma^{\star, I I}$, which pulls these orbitals more strongly inside the nucleus. At the same time both choices yield the same spectroscopic factors, implying equal occupancies. The depletion of these orbits is driven by the coupling to long-range collective excitations contained in $\Sigma^{(2 \mathrm{plh}) \text {, Fadd }}$ and $\Sigma^{(2 \mathrm{hlp}) \text {,Fadd }}$. An additional quenching is expected from short-range and tensor correlations and was not accounted for in this work. This has been seen to be of about $10 \%$ for bound orbitals of several closed shell nuclei [10]. However, what the strength

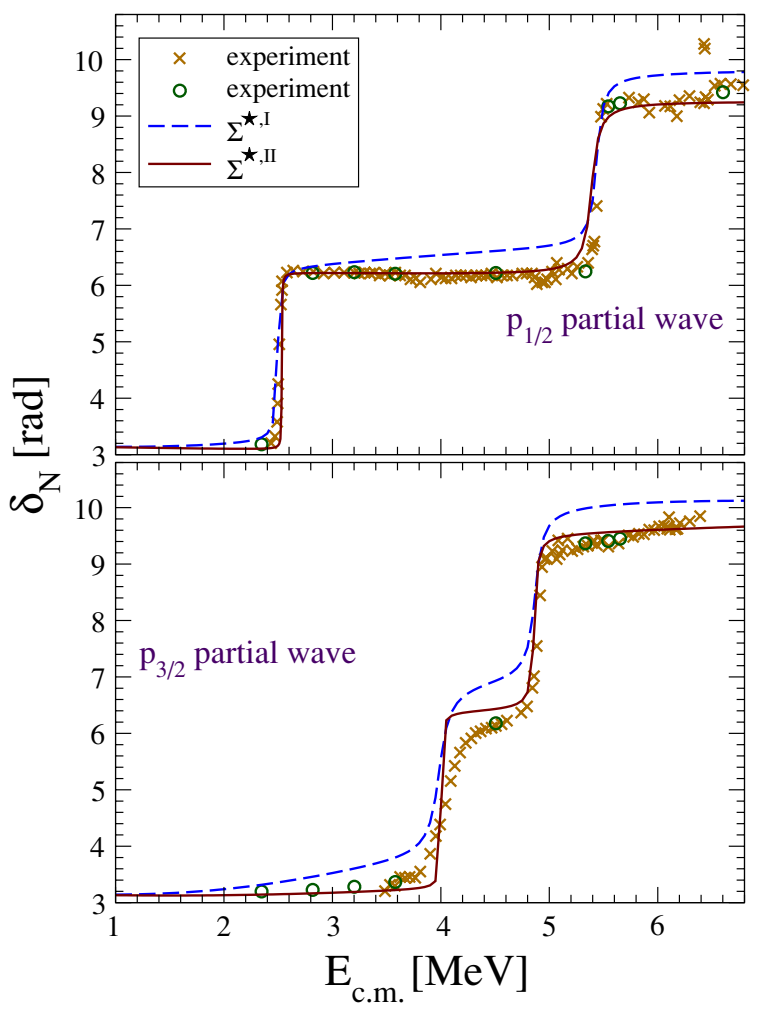

FIG. 5. (Color online) Phase shifts for negative parity waves obtained from the the self-energies I (dashed lines) and II (full lines). The experimental results are from Refs. [31] (crosses) and [32] (circles).

of this reduction should be for loosely bound nucleons, which can be largely localized at radii outside the nuclear surface, has not yet been investigated.

\section{DISCUSSION}

In order to include the principals physics ingredients, an improved self-energy was constructed from those obtained by two different calculations of the nucleus of ${ }^{16} \mathrm{O}$. The effects of coupling a nucleon to collective modes were studied in Ref. [14] but in a restricted model space. The momentum components of the mean field potential outside this space were instead extracted from Ref. [16]. Since these calculations

TABLE II. Corrections applied to the depth of the MF potentials I and II [Eq. (3)] for the calculation of Figs. 4 and 5 .

\begin{tabular}{lccc}
\hline \hline$l j$ & $\Sigma^{\star, I}$ & $\Sigma^{\star, I I}$ & $E_{\mathrm{c} . \mathrm{m} .}^{\exp }(\mathrm{MeV})$ \\
& $N_{l j}^{I \mathrm{a}}$ & $N_{l j}^{I I \mathrm{a}}$ & \\
\hline$d_{3 / 2}$ & 1.08 & 1.24 & 4.4 \\
$s_{1 / 2}$ & 1.05 & 1.24 & -0.1 \\
$d_{5 / 2}$ & 1.16 & 1.29 & -0.6 \\
$p_{1 / 2}$ & 1.06 & 1.15 & -12.1 \\
$p_{3 / 2}$ & 1.07 & 1.25 & -18.5 \\
\hline \hline
\end{tabular}

${ }^{\mathrm{a}} E_{\mathrm{c} . \mathrm{m} .}^{l j} \equiv E_{\mathrm{c} . \mathrm{m} .}^{\mathrm{exp}}$, except when specified. 


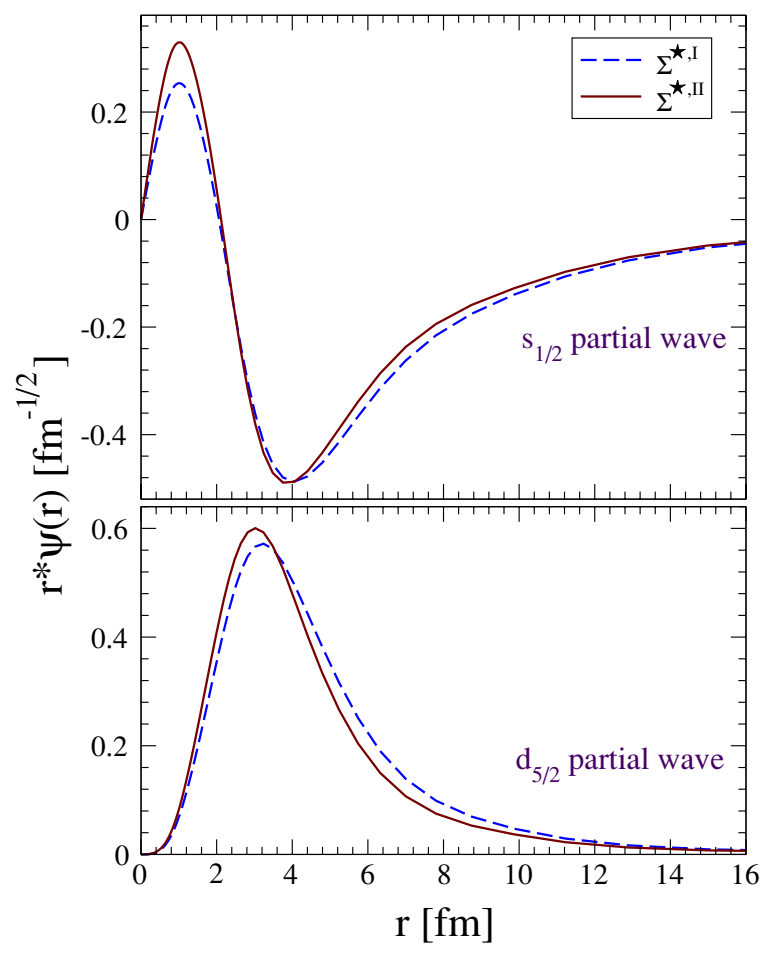

FIG. 6. (Color online) Radial part of the overlap wave functions between ${ }^{16} \mathrm{O}$ and the bound $d_{5 / 2}$ and $s_{1 / 2}$ states of ${ }^{17} \mathrm{~F}$.

are based on realistic two-body internucleon reactions, the energy spectra cannot be accurately reproduced. Therefore, the model has to be constrained phenomenologically to reproduce the experimental spectra of the nuclei with $A \pm 1$ nucleons. Resolving this situation may require the use of multinucleon forces and more appropriate effective interactions.

The present results show that both the inclusion of all momentum components of the particle-hole Hilbert space and a proper treatment of long-range correlations are important to correctly reproduce the mean field optical potential. The coupling of single particle strength to long-range excitations is also responsible for the creation of non-mean-field resonances. After constraining the prediction for the single particle energies, the phase shifts for the scattering of protons from ${ }^{16} \mathrm{O}$ were obtained in fair agreement with the experimental data, except for the background behavior of the of the $p$ waves. The difficulties for these waves are accompanied by the issue of explaining the hole spectroscopic factors with the same parity extracted from $\left(e, e^{\prime} p\right)$ experiments [34]. In Ref. [14] the latter were linked to the particular structure of the low-energy spectrum of ${ }^{16} \mathrm{O}$ and further studies along this line
TABLE III. Spectroscopic factors, ANCs (in $\mathrm{fm}^{-1 / 2}$ ) and rootmean-square radii (in fm) for the bound $d_{5 / 2}$ and $s_{1 / 2}$ orbitals of ${ }^{17} \mathrm{~F}$.

\begin{tabular}{lccccccc}
\hline \hline & \multicolumn{4}{c}{$s_{1 / 2}$} & & & \multicolumn{3}{c}{$d_{5 / 2}$} \\
\cline { 2 - 3 } \cline { 6 - 8 } & $Z_{s_{1 / 2}}$ & $C_{s_{1 / 2}}$ & $\left\langle r_{s_{1 / 2}}^{2}\right\rangle^{1 / 2}$ & & $Z_{d_{5 / 2}}$ & $C_{d_{5 / 2}}$ & $\left\langle r_{d_{5 / 2}}^{2}\right\rangle^{1 / 2}$ \\
\hline$\Sigma^{\star, I}$ & 0.931 & -82.5 & 5.86 & & 0.913 & 1.07 & 4.01 \\
$\Sigma^{\star, I I}$ & 0.921 & -73.9 & 5.55 & & 0.909 & 0.81 & 3.70 \\
\hline \hline
\end{tabular}

have been initiated in Ref. [12]. It is plausible that the required improvements will resolve both the problems of spectroscopic factors and scattering phase shifts. We note that similar issues are expected to be beyond the requirements for reproducing most heavier closed shell nuclei $[28,35]$. Thus, the study of ${ }^{16} \mathrm{O}$ can be seen as a stringent test case for the present approach.

\section{CONCLUSIONS}

This work investigates the possibility of describing nucleon-nucleus scattering employing the many-body selfenergy as an optical potential. This corresponds to applying the Feshbach projection formalism to an Hilbert space containing both particle and hole states.

The present results are also a first attempt at computing scattering processes using the many-body Green's functions and required to introduce specific phenomenological corrections to reproduce the single particle spectrum. However, it is shown that predictions for the scattering of nucleons can be obtained working also in the particle-hole space. The present work gives insight into the developments that will be needed to pursue reliable microscopic calculations of the optical potential. We feel that the overall quality of the results can be comparable to other methods applicable at low energies [36,37], when the missing ingredients that are needed to reproduce the resonance spectrum are included. Thus, SCGF theory could be considered a valid candidate for the study of selected reactions at astrophysical energies.

\section{ACKNOWLEDGMENTS}

It is a pleasure to acknowledge several useful discussions with J.-M. Sparenberg and W. H. Dickhoff. We also thank Professor Dickhoff for providing his results form Ref. [16]. We thank the Institute for Nuclear Theory at the University of Washington for its hospitality during the initial writing of the manuscript. This work was supported by the Natural Sciences and Engineering Research Council of Canada (NSERC).
[1] Feshbach, Ann. Phys. (NY) 5, 357 (1958); 19, 287 (1962).

[2] R. G. Lovas, R. J. Liotta, A. Insolia, K. Varga, and D. S. Delion, Phys. Rep. 294, 265 (1998).

[3] K. Amos, P. J. Dortmans, H. V. von Geramb, S. Karataglidis, and J. Raynal, Adv. Nucl. Phys. 22, 275 (2000).
[4] J. Okołowicz, M. Płoszajczak, and I. Rotter, Phys. Rep. 374, 271 (2003).

[5] F. Capuzzi and C. Mahaux, Ann. Phys. (NY) 245, 147 (1996).

[6] J. Escher and B. K. Jennings, Phys. Rev. C 66, 034313 (2002). 
[7] A. L. Fetter and J. D. Walecka, Quantum Theory of ManyParticle Physics (McGraw-Hill, New York, 1971).

[8] A. A. Abrikosov, L. P. Gorkov, and I. E. Dzyaloshinski, Methods of Quantum Field Theory in Statistical Physics (Dover, New York, 1975).

[9] C. Mahaux and R. Sartor, Adv. Nucl. Phys. 20, 1 (1991).

[10] W. H. Dickhoff and C. Barbieri, Prog. Part. Nucl. Phys. 52, 337 (2004).

[11] C. Barbieri and W. H. Dickhoff, Phys. Rev. C 63, 034313 (2001).

[12] C. Barbieri and W. H. Dickhoff, Phys. Rev. C 68, 014313 (2003).

[13] P. Danielewicz and P. Schuck, Nucl. Phys. A567, 78 (1994).

[14] C. Barbieri and W. H. Dickhoff, Phys. Rev. C 65, 064313 (2002).

[15] C. Barbieri and B. K. Jennings, Proceedings of the Eight Symposium of Nuclei in the Cosmos, July 2004, nucl-th/0408017 (to be published in Nucl. Phys. A).

[16] H. Müther and W. H. Dickhoff, Phys. Rev. C 49, R17 (1994); H. Müther, A. Polls, and W. H. Dickhoff, ibid. 51, 3040 (1995).

[17] J. Winter, Nucl. Phys. A194, 535 (1972).

[18] W. J. W. Geurts, K. Allaart, and W. H. Dickhoff, Phys. Rev. C 50, 514 (1994).

[19] R. Machleidt, Adv. Nucl. Phys. 19, 191 (1989).

[20] H. Müther and P. U. Sauer, in Computational Nuclear Physics, edited by K.-H. Langanke, J. A. Maruhn, and S. E. Koonin (Springer, New York, 1993).

[21] A. Polls, M. Radici, S. Boffi, W. H. Dickhoff, and H. Müther, Phys. Rev. C 55, 810 (1997).
[22] Y. R. Kwon and F. Tabakin, Phys. Rev. C 18, 932 (1978).

[23] C. M. Vincent and S. C. Phatak, Phys. Rev. C 10, 391 (1974).

[24] S. C. Pieper and R. B. Wiringa, Annu. Rev. Nucl. Part. Sci. 51, $53(2001)$

[25] P. Navrátil and E. W. Ormand, Phys. Rev. C 68, 034305 (2003).

[26] H. Müther, R. Machleidt, and R. Brockmann, Phys. Rev. C 42, 1981 (1990); S. C. Pieper and V. R. Pandharipande, Phys. Rev. Lett. 70, 2541 (1993).

[27] D. J. Dean, T. Engeland, M. Hjorth-Jensen, M. Kartamyshev, and E. Osnes, Prog. Part. Nucl. Phys. 53, 419 (2004).

[28] M. G. E. Brand, K. Allaart, and W. H. Dickhoff, Nucl. Phys. A509, 1 (1990).

[29] J. Yuan, Ph.D. thesis, Washington University, St. Louis, 1994.

[30] P. Bhattacharyya et al., Phys. Rev. Lett. 87, 062502 (2001).

[31] S. R. Salisbury and H. T. Richards, Phys. Rev. 126, 2147 (1962).

[32] R. A. Blue and W. Haeberli, Phys. Rev. B 137, 284 (1964).

[33] C. Barbieri (unpublished).

[34] M. Leuschner et al., Phys. Rev. C 49, 955 (1994).

[35] G. A. Rijsdijk, K. Allaart, and W. H. Dickhoff, Nucl. Phys. A550, 159 (1992).

[36] D. Baye, P. Descouvemont, and M. Hesse, Phys. Rev. C 58, 545 (1998).

[37] K. Bennaceur, F. Nowacki, J. Okołowicz, and M. Płoszajczak, Nucl. Phys. A671, 203 (2000).

[38] The isospin degrees of freedom are not shown explicitly here. 